\title{
Hybrid MPC-Based Automatic Generation Control for Dominant Wind Energy Penetrated Multisource Power System
}

\author{
Naimul Hasan (D, ${ }^{1}$ Ibrahim Alsaidan $\left(\mathbb{D},{ }^{1}\right.$ Mohammad Sajid $\mathbb{D}^{2},{ }^{2}$ Shahida Khatoon, ${ }^{3}$ \\ and Shuaib Farooq ${ }^{3}$ \\ ${ }^{1}$ Department of Electrical Engineering, College of Engineering, Qassim University, Buraydah, Saudi Arabia \\ ${ }^{2}$ Department of Mechanical Engineering, College of Engineering, Qassim University, Buraydah, Saudi Arabia \\ ${ }^{3}$ Department of Electrical Engineering, Jamia Millia Islamia, New Delhi, India
}

Correspondence should be addressed to Naimul Hasan; na.hasan@qu.edu.sa

Received 27 January 2021; Revised 21 November 2021; Accepted 16 December 2021; Published 17 January 2022

Academic Editor: Michele Calì

Copyright ( 2022 Naimul Hasan et al. This is an open access article distributed under the Creative Commons Attribution License, which permits unrestricted use, distribution, and reproduction in any medium, provided the original work is properly cited.

\begin{abstract}
This paper presents hybrid model predictive control-based automatic generation regulator design for dominant wind energy penetrated multisource power system. The other power generation sources hydro and thermal are also considered in each area. The proposed AGC regulator is designed for each independent area considering only local power system states to ensure the stability of closed-loop system under small perturbation. The performance of the proposed AGC regulator has been validated for $2 \%$ step load perturbation. Furthermore, system parameter is varied to $20 \%$ of nominal value to assess the robust performance of the regulator. Performance evaluation between the hybrid MPC, conventional MPC, and traditional AGC regulators considering the dominant participation of the DFIG wind turbines is presented to demonstrate the superior performance of the hybrid MPC AGC regulator.
\end{abstract}

\section{Introduction}

The automatic generation control is an important aspect of regulating the frequency by controlling the real power generation in a large interconnected power system. It is fundamentally responsible for maintaining frequency and scheduled power flow over the tie-lines. Traditionally, frequency regulation is done in a hierarchical structure of controls installed at different levels. The primary and secondary control is often PI-based, which is tuned with operator experience. And it works in automatic mode; however, tertiary control is manually controlled by the operator at the dispatch center. The secondary control is often referred to as automatic generator control (AGC). The role of AGC in unit commitment is also very important for the steady-state operation of the power system, and problem formulation is considered in mixed integer nonlinear programming method subject to frequency limits and generation rate constraints.

The AGC is coupled with the unit commitment schedule rather than sequential scheduling of reserves according to contingent generation levels $[1,2]$. Several AGC schemes have been reported to study the dynamic stability of power system subject to different disturbance conditions. These schemes range from conventional droop control to various modern optimal control strategies. The conventional controller gives a better dynamic response, but the performance degrades as the system grows and utilizes multiple generation sources. Thus, for a large network, the modern controllers are more reliable and afford the flexibility in accordance to the power system structure. Model predictive control (MPC) is one of the other viable solutions for the AGC problem and has received considerable attention since its invention. Due to the communication limitations of MPC for geographically distributed control areas, distributed model predictive control (MPC) strategies for controlling largescale power systems are a viable solution of communication issues [3-5]. The overall system is divided into subsystems, and each subsystem is equipped with MPC controller. The predictive scheme for nonlinear multivariable power plant control is presented in [6]. A load frequency control (LFC) 
design using the model predictive control (MPC) technique [7] in a multiarea power system in the presence of wind turbines is presented, and each local area controller is designed independently such that stability of the overall closed-loop system is guaranteed.

Shiroei et al. presented the functional model predictive control and robust multivariable-based AGC scheme with generation rate constraint $[8,9]$. The modified model predictive control objective function that includes generation rate constraints and economic dispatch is discussed in [9]. In paper [10], the authors proposed to utilize the mixing of adaptive model predictive controller (AMPC) and recursive polynomial model estimator (RPME) integrated with double-fed induction generator wind turbines for frequency control system. The researchers [11] presented the work that uses the reduced order model having dominant modes of frequency oscillation is identified which is updated by predictive control for optimal control in each window. The authors [12] focused on the application of the heat pump water heater (HPWH) for secondary frequency control and applied model predictive control (MPC) method for a twoarea power system.

The supervisory predictive control for frequency regulation, which computes the optimal set-points for decentralized local controllers for smart grid comprising of wind and solar power plant, is presented in [13]. The MPCbased AGC scheme is presented in [14] for Nordic power system considering generation rate constraint, limitations on tie-line power flow, and generation capacity with the participation factors as optimization variables. An effective hybrid of distributed model predictive control and fuzzy logic, which is used to limit the governor valve, is incorporated in AGC scheme for multiarea hydrothermal system considering the nonlinearity [15]. A fuzzy adaptive model predictive scheme performance is quite appreciable for frequency control of a microgrid having generating sources of controllable and uncontrollable nature [16]. The authors [17] proposed the work on an adaptive artificial fuzzy Mamdani-based model predictive approach for load frequency regulation of an isolated microgrid. The authors considered conventional model predictive approach equipped by a fuzzy logic tuning mechanism. In paper [18], the authors proposed the modified Jaya optimization algorithm (MJOA) by considering a weight parameter in the search process for online tuning the controller parameters of automatic generation control (AGC) of wind integrated power system. The MPC has efficient capability of handling the constraints, and MPC scheme which includes economic logic for cost reduction is presented in [19-21].

A good number of studies reported the supervisory role of model predictive controller, which gives optimum response considering the communication delays, nonlinearity, and coordination from distributed controllers. The work presented $[22,23]$ on the frequency support from penetration of the wind energy and improvement interarea oscillation damping. The authors [24] proposed the intelligent predictive controller combined with predictive control and neural network for the design of AGC controller for thermal power units. The authors proposed a Bayesian neural net- work identification model for typical ultra super critical thermal power unit. In paper [25], LFC synchronized with AVR in three-area interconnected power system is proposed. Model predictive controller (MPC) is configured in a dense distributed pattern due to its online set-point tracking, and it is used as the supplementary controller. The paper [26] presents the work on hybrid Taguchi-genetic algorithm (HTGA) tuned with automatic generation controller for two areas interconnected multisource power system model. Taguchi method which is based on modified statistical approach and has systematic reasoning ability is used to enhance the performance of genetic algorithm. The authors [27] presented the work on a novel ETC architecture design for load frequency control (LFC) with supplementary adaptive dynamic programming (ADP). The authors [28] proposed the frequency control mechanism for renewable energy-based smart grid using adaptive control techniques and model predictive control (MPC) based on the multi objective practical swarm optimization (MOPSO) algorithm.

The authors [29] presented the work on designing of a model predictive controller for the application of automatic generation control of a two-area interconnected power system consisting of thermal, hydro, and wind power generation units. The comparative analysis is done with conventional classical PI and PID automatic generation controllers. The authors [30] introduced a model predictive controller for load frequency control of an isolated ac microgrid system, clustered with the wind system and solar system and plugged in an electric vehicle. The work is also presented on model predictive controller for microgrid load frequency control. In paper [31], the authors presented the work on enhancement of the output response of automatic voltage regulator using model predictive controller and the proposed technique is compared with the proportionalintegral-derivative (PID) controller tuned with the genetic algorithm (GA).

This paper presents a distributed hybrid MPC-based AGC regulator for dominant wind energy penetrated twoarea multisource power system. Each area has $60 \%$ participation of DFIG wind power in frequency regulation whereas the remaining $40 \%$ is contributed by the thermal and hydropower plant. The control output of each distributed MPC and fixed structure PID is based on the area control error, which is merely calculated from the disturbed system states of each control area. The performance of the proposed hybrid MPC technique is tested for $2 \%$ load perturbation in area-1 after which the effect of parameter variation of about $20 \%$ of nominal for the same load disturbance is also verified. Furthermore, the performance of proposed AGC scheme is also compared with MPC and fixed structure PID controller to evaluate the proposed AGC scheme. The simulation study also established the superiority in performance of the proposed AGC scheme over the other conventional AGC schemes for DFIG dominant multisource power system. Since fixed structured PID controller is widely used in power industry for the load frequency control application but due to the integration of renewable plant in the grid, the performance of the PID degrades which in turn fails to 


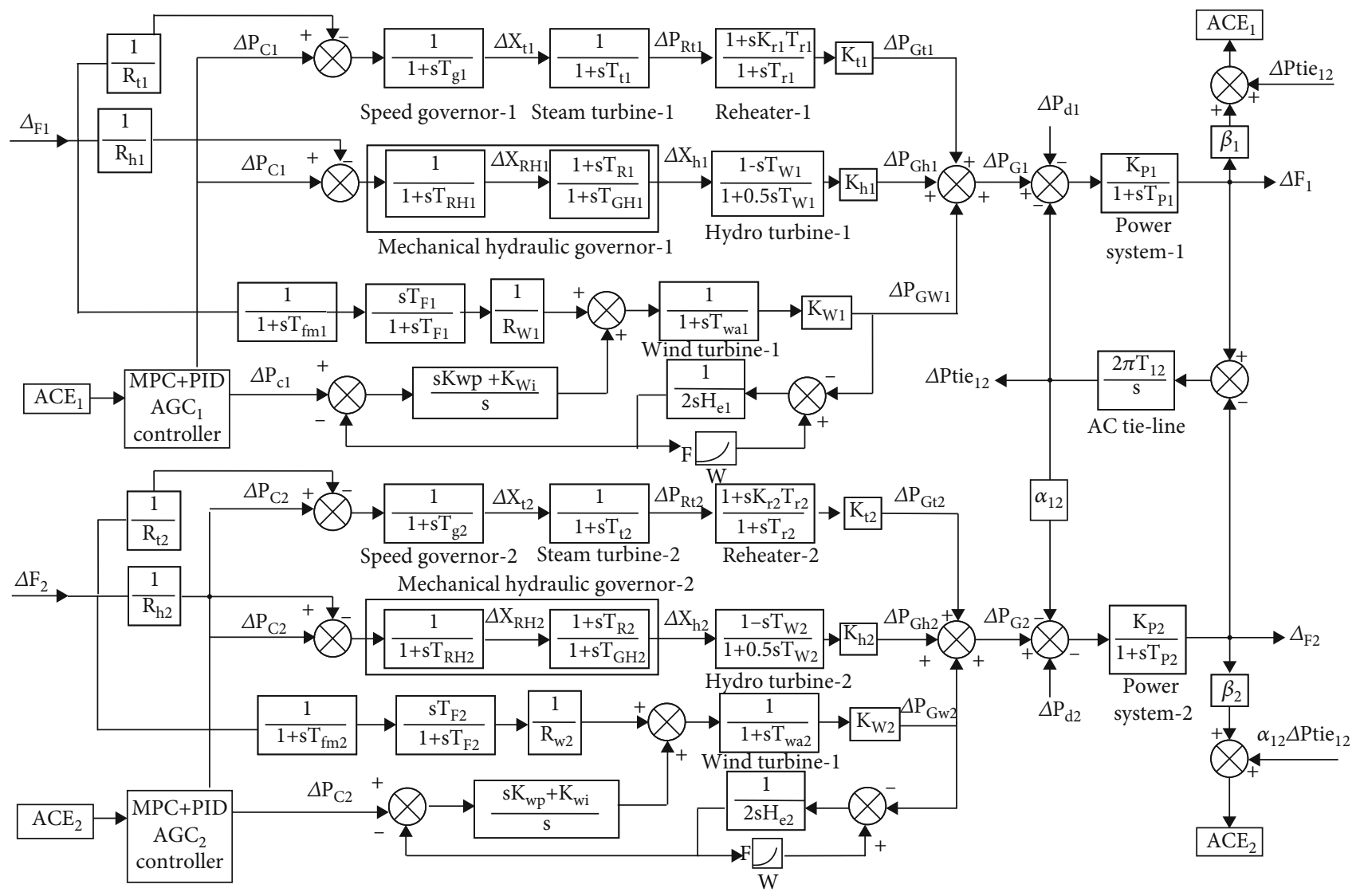

FIGURE 1: Transfer function model of multisource power system for MPC+PID AGC study.

control the frequency of the control area in an efficient way. Therefore, in this paper, the performance of PID controller is evaluated by hybridizing the model predictive control. The novelty of the work is that MPC is evaluated as performance enhancer for the PID controller, and the hybrid controller is able to handle the uncertainties more efficiently of the multisource power system. The paper is organized as follows: two-area multisource power system model is presented in Section 2. The description of hybrid MPC with objective function for multisource power system is given in Section 3. The simulation results and discussions on proposed hybrid AGC, conventional PID, and traditional MPC scheme are described in Sections 4 and 5. Finally, the conclusion of the present work is presented in Section 6.

\section{Power System Model for Hybrid MPC- AGC Study}

The composite transfer function model of two-area interconnected multisource power system to study the proposed AGC scheme is shown in Figure 1. The model consists of thermal, hydro, and DFIG power plants. The control area is interconnected and exchanging the scheduled power between the areas via ac tie-line. The interconnected areas increase the system reliability through generation demand balance, in case of any load perturbation or the trip of generating units. The linear continuous-time dynamic system can be represented by the standard state space model as follows:

$$
\begin{aligned}
\frac{d}{d t}(X) & =A X+B U+\Gamma P_{d} \\
Y & =C X
\end{aligned}
$$

where the matrices and vectors are represented for system, input, disturbance, and output, respectively, in standard nomenclatures.

In this paper, a decentralized MPC approach is presented; therefore, the two-power system model is segregated into independent areas, and the corresponding differential equation for area-1 is derived to design the decentralized MPC for each area.

$$
\begin{aligned}
\frac{d}{d t}\left(x_{1}\right)= & -\frac{1}{T_{P 1}} x_{1}-\frac{K_{P 1}}{T_{P 1}} x_{2}+\frac{K_{P 1}}{T_{P 1}} x_{5} \\
& +\frac{K_{P 1}}{T_{P 1}} x_{8}-\frac{K_{P 1}}{T_{P 1}} \Delta P_{d 1}, \\
\frac{d}{d t}\left(x_{2}\right)= & -\frac{1}{T_{r 1}} x_{2}+\left(\frac{1}{T_{r 1}}-\frac{K_{r 1}}{T_{t 1}}\right) x_{3}+\frac{K_{r 1}}{T_{r 1}} x_{4},
\end{aligned}
$$




$$
\begin{aligned}
& \frac{d}{d t}\left(x_{3}\right)=-\frac{1}{T_{t 1}} x_{3}+\frac{1}{T_{t 1}} x_{4}, \\
& \frac{d}{d t}\left(x_{4}\right)=-\frac{1}{R_{1} T_{g 1}} x_{1}-\frac{1}{T_{g 1}} x_{4}+\frac{1}{T_{g 1}} \Delta P_{C 1}, \\
& \frac{d}{d t}\left(x_{5}\right)=\frac{1}{0.5 T_{G H 1} R_{h 1}} x_{1}-\frac{1}{0.5 T_{w 1}} x_{5} \\
& +\left(\frac{1}{0.5 T_{w 1}}+\frac{K_{r 1}}{0.5 T_{G H 1}}\right) x_{6} \text {, } \\
& \frac{d}{d t}\left(x_{6}\right)=-\frac{1}{R_{h 1} T_{G H 1}} x_{1}-\frac{1}{T_{G H 1}} x_{6}+\frac{1}{T_{G H 1}} \Delta P_{C 1} \text {, } \\
& \frac{d}{d t}\left(x_{7}\right)=-\frac{1}{R_{h 1} T_{R H 1}} x_{1}-\frac{1}{T_{R H 1}} x_{7}+\frac{1}{T_{R H 1}} \Delta P_{C 1} \text {, } \\
& \frac{d}{d t}\left(x_{8}\right)=-\frac{1}{T_{w a 1}} x_{8}+\frac{1}{R_{w 1} T_{w a 1}} x_{10}+\frac{1}{T_{w a 1}} x_{12} \text {, } \\
& \frac{d}{d t}\left(x_{9}\right)=\frac{1}{T_{f m 1}} x_{1}-\frac{1}{T_{f m 1}} x_{9}, \\
& \frac{d}{d t}\left(x_{12}\right)=-K_{\omega i 1} x_{11}+\Delta \omega_{\text {ref } w 1} K_{\omega i 1}, \\
& \frac{d}{d t}\left(x_{13}\right)=\beta_{1} x_{1} \text {. }
\end{aligned}
$$

The state space model is obtained using Equations (3)-(13). Therefore, for area-1, the order of state matrix $[A$ ] is $13 \times 13$, the order of control matrix $[B]$ is $13 \times 1$, disturbance matrix $[\Gamma]$ is $13 \times 1$, and the output matrix $[C]$ is an identity matrix of appropriate dimensions. The disturbance in one area affects the frequency and tie-line power, which is defined as area control error. Below is the mathematical formulation of area control error.

$$
\begin{aligned}
& \mathrm{ACE}_{1}=\Delta P_{\text {tie }}+B_{1} \Delta f_{1}, \\
& \mathrm{ACE}_{2}=\alpha_{12} \Delta P_{\text {tie }}+B_{2} \Delta f_{2},
\end{aligned}
$$

where $B$ is tie-line bias constant and $\alpha_{12}$ is the area size ratio. The AGC schemes are developed and implemented to regulate area control error (ACE) for the stable operation under the steady-state condition. The symbols and abbreviation are having their usual presentation [26].

\section{Hybrid MPC-Based Automatic Generation Controller}

The hybrid MPC is a combination of MPC and PID controller. Each controller is designed independently for the multisource interconnected system. The area control error (ACE) signal is used as the input to controller in both the cases. The control signal is calculated on the basis of area control error $\left(\mathrm{ACE}_{i}\right)$, and the reference value of $\mathrm{ACE}_{\text {ref }}$ for steady state is zero. Finally, the output of model predictive control and PID is algebraically added for regulating the frequency of the dis-

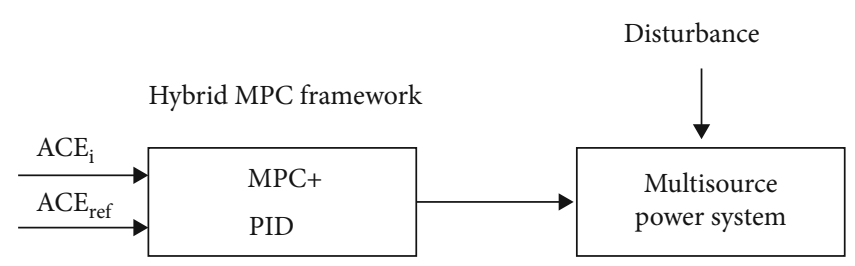

FIGURE 2: Block diagram of hybrid MPC for AGC.

turbed area. The hybrid MPC framework is shown in Figure 2.

3.1. Model Predictive Control. MPC has seen widely used in chemical and petrol industries for a variety of control applications. It utilizes a system's prediction model to obtain the control behavior after the minimization of a defined objective function. Moreover, constraint on input and output is also considered while optimizing the response of predicted model. For the prediction model to output accurately, the model should represent its dependency on future input and current output. Therefore, optimization problem is duly set up by capturing plant behavior. The input is fed to the plant in an optimal sequence at each control interval, and the subsequent output is measured which is then fed to optimization solver to obtain the desired control input to the plant. The procedure is repeated at the subsequent time intervals with fresh measurements from plant. Therefore, it is very important to solve optimization problem within the designed time frame. However, failure in the optimization on time can lead to instability in controlled plant. Therefore, MPC is conventionally used for slow dynamic processes having an adequate time for solving the optimization problem. Mathematically, MPC is based on the state space linear state space model.

$$
\begin{gathered}
x(k+1)=A x(k)+B_{u} u(k)+B_{v} v(k)+B_{d} d(k), \\
y(k)=C x(k)+D_{v} v(k)+D_{d} d(k),
\end{gathered}
$$

where $x(k)$ is system state, $u(k)$ is control state, $v(k)$ is measured disturbances, $d(k)$ is unmeasured disturbances, and $y$ $(k)$ is the output vector. The $A, B, C$, and $D$ matrices are the corresponding matrices of the system. At each time instant, the MPC algorithm takes the measurement of the output, reference, and disturbance. Based on the last control and state, the current state is estimated.

$$
\begin{aligned}
& \hat{y}=C_{m} \hat{x}_{0}+D_{v m}, \\
& \hat{x}=\hat{x}_{0}+L(y-\hat{y}),
\end{aligned}
$$

where $L$ is the estimator gain, $y$ is the output, and $y^{\wedge}$ is the estimated output. The state estimate is here denoted as $x^{\wedge}$. $\mathrm{Cm}$ is the estimated state matrix, and $D_{v m}$ is measured disturbances. All the values are initialized before the output is computed. 
The output sequence was calculated up to $N$ steps, minimizing the objective function which is given as follows:

$$
\begin{gathered}
\Delta U=[u(t), u(t+1), . . \cdots, u(t+N)], \\
\min _{x(t), u(t)} J[x(t), u(t)],
\end{gathered}
$$

subject to constraints

$$
g(x(t)), u(t) \leq 0
$$

where $J$ is the objective function solved over a fixed time horizon and $x(t)$ and $u(t)$ are system states and controlled states, respectively. The MPC solves an optimization problem within a fixed time frame pertaining to the objective functions. The control loop is closed by a system measurement. It is considered that the initial state vector as well as predicted system disturbance is known, and the system measurement ensures the closed-loop control.

The decentralized model predictive control is designed here for automatic generation control, which has a benefit of shorter optimization time and is based on states of each control area of an interconnected multisource model. The control objectives for desired controller are frequency regulation within safe limits and the minimum deviation of tie-line power from its scheduled values. The generalized form of the objective function solved by the MPC is given as follows:

$$
J(x, u, \varepsilon)=\int_{t=0}^{T} x^{T} Q x+\left(u-u_{0}\right)^{T} R\left(u-u_{0}\right)+\gamma \varepsilon d t .
$$

$Q$ and $R$ are the tuning matrices and have their usual abbreviation. The objective function is derived from area control error and output of controller. The state space formulation of the objective function for the MPC is given in Equation (24).

$$
\begin{aligned}
\underset{\Delta u}{\min J[k]} & =\sum_{i=1}^{m}\left[\left(F_{T}+P_{T}+U_{T}\right]+\gamma \mathcal{\varepsilon},\right. \\
P_{T} & =(P[k+i]-P[k]) Q_{p}(P[k+i]-P[k])^{\prime}, \\
F_{T} & =(f[k+i]-f[k]) Q_{f}(f[k+i]-f[k])^{\prime}, \\
U_{T} & =(u[k+i]-u[k]) Q_{u}(u[k+i]-u[k]),
\end{aligned}
$$

where $f$ is the system frequency, $P$ is the tie-line power, $Q_{f}, Q_{p}$, and $Q_{u}$ are the penalty on frequency, tie-line power, and output, respectively, $Y$ is used for tuning, and $\varepsilon$ is the slack positive variable added to the output constraint and used to avert nonfeasible solutions by softening few states of the system. The control consists of the predicted control signals for $N_{c}$ steps ahead and calculated in every step.

$$
\Delta U=r-K \hat{x}
$$

where $r$ is the reference and $x^{\Lambda}$ is the estimated state. Here, the control horizon $N_{c}$ is selected less than the prediction horizon
TABLE 1: Optimal gain of PID and performance indices.

\begin{tabular}{lcccc}
\hline Gain & & & & \\
Tuning method & $K p$ & $K i$ & $K d$ & ISE \\
\hline Conventional & 0.14681 & 0.59983 & 0.34684 & $8.4477 \times 10^{-4}$ \\
HTGA & 1.41610 & 1.01623 & 1.08543 & $4.355 \times 10^{-4}$ \\
\hline
\end{tabular}

TABLE 2: Optimal parameters of MPC and performance indices.

\begin{tabular}{lccc}
\hline $\begin{array}{l}\text { Parameters } \\
\text { Tuning method }\end{array}$ & Prediction horizon & Control horizon & ISE \\
\hline Manual iteration & 14 & 8 & 0.34684 \\
HTGA & 10 & 5 & 1.08543
\end{tabular}

$N_{p}$ due to control trajectory without having the predicted states at that instant.

$$
N_{c}<N_{p}
$$

For the optimal tuning of conventional PID AGC regulator, the optimization goal is for minimizing objective function which is formulated as square of deviation in frequency over time and is given as below:

$$
J_{\min }=\int_{0}^{t}\left(\Delta f_{i}^{2}\right) d t
$$

where $f_{i}$ is the frequency of the $i^{\text {th }}$ area.

\section{Simulation Study}

The simulation work is carried out for the proposed AGC controller having the combined structure of model predictive and proportional integral derivative controller. The total power generated in each area is contributed by DFIG wind turbine, thermal, and hydro with a participation factor of $60 \%, 30 \%$, and $20 \%$, respectively. Thus, the impact of DFIG-based wind generating units in AGC regulation scheme is dominant. The efficacy of the proposed scheme is validated by the dynamic performance of the system considering area-1 load perturbation of $2 \%$.

The gain of the PID is tuned using the hybrid Taguchigenetic algorithm [26]. It is used for solving global optimization problems with continuous variables. The optimal gain obtained by HTGA is used in the proposed hybrid MPC controller for AGC is given in Table 1.

The parameter of the MPC controller such as prediction and control horizon is considered as 10 and 5, respectively, for simulation (Table 2). The sampling time is taken as $0.2 \mathrm{~s}$.

The constraints used in the simulation study are generation rate constraints, thermal plant $|\Delta P t i| \leq 0.0017 \mathrm{puMW} / \mathrm{s}$, and hydroplant $|\Delta P h i| \leq 0.045 \mathrm{puMW} / \mathrm{s}$.

4.1. Performance of Hybrid MPC-Based AGC Scheme. The system performance is investigated with the proposed AGC scheme for change of $0.02 \mathrm{pu}$ at $10 \mathrm{~s}$ in area-1 step load. The effectiveness of the proposed scheme is evaluated by comparing the results with fixed structure proportional- 

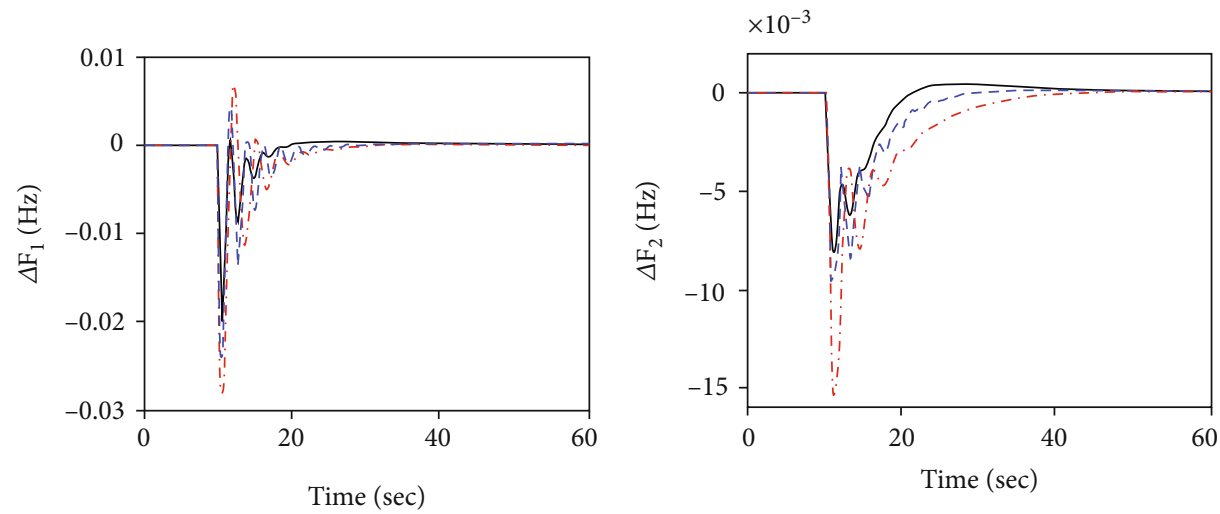

... AGC with PID

- - AGC with MPC

... AGC with PID

- - AGC with MPC

— AGC with MPC+PID

(a)

(b)
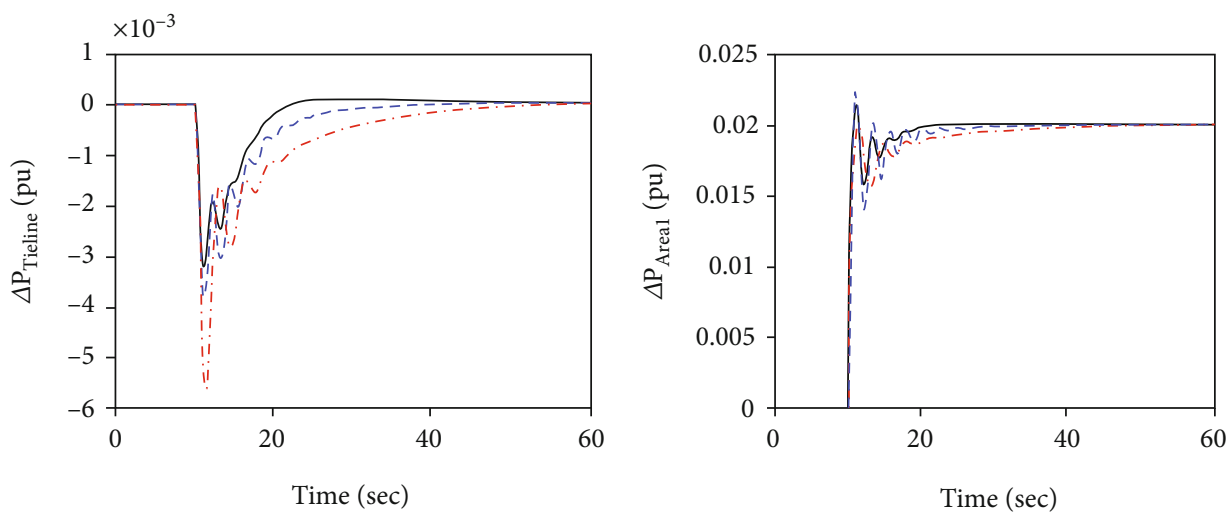

... AGC with PID

- - - AGC with MPC

- - - AGC with PID

-. AGC with MPC

— AGC with MPC+PID

(c)

(d)

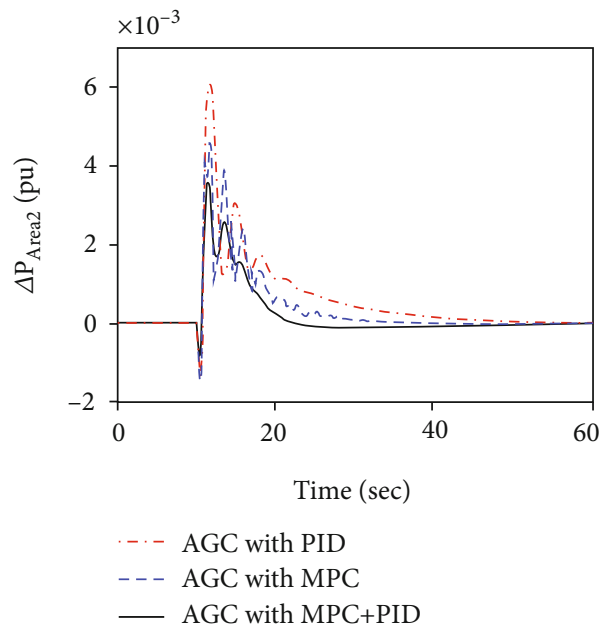

(e)

Figure 3: Dynamic performance of multisource power system model for $2 \%$ load disturbance in area-1. (a) $\Delta F_{1}$ vs. time, (b) $\Delta F_{2}$ vs. time, (c) $\Delta P_{\text {tie }}$ vs. time, (d) $\Delta P_{\text {area-1 }}$ vs. time, and (e) $\Delta P_{\text {area-2 }}$ vs. time. 
integral-derivative (PID) and model predictive-based AGC scheme. Figure 3(a) depicts the frequency response of area1 for the conventional PID, MPC, and MPC+PID-based automatic generation controller for two-area interconnected system comprising of multisource. From first sight of inspection, it is quite clear that the MPC+PID-based AGC scheme improves the peak deviation by $50 \%$ and $23 \%$, respectively, over the fixed structure conventional PID and MPC-based AGC scheme. Similarly, the settling time is also improved by $50 \%$ and $10 \%$, respectively, over the conventional schemes. Moreover, due to the presence of weak tie-line between the control areas, a similar dynamic is also observed in area-2 as shown in Figure 3(b), inferring the good performance of proposed AGC scheme in other area too.

The tie-line power dynamics is displayed in Figure 3(c), which also clearly indicates the performance improvement by approximate $52 \%$ and $35 \%$ in peak deviation as well as $50 \%$ and 20\% improvement in settling time. Furthermore, the oscillations are also drastically damped out by the MPC +PID-based AGC scheme. Depending on the AGC scheme, the dynamics of power generation in different sources is also regulated with respect to their contribution in total power generation in their respective area. Due to load perturbation in area-1, the total power generated is also changed to regulate the frequency to its nominal value. The corresponding dynamics of total power generated in area- 1 and area- 2 by thermal, hydro, and wind unit is shown in Figures 3(d) and 3(e), respectively.

From the inspection of responses of different generating units in area-1, it is clearly visible that the power regulation of thermal, hydro, and wind has reduced peak deviation and improved settling time with very low oscillations in frequency and power. Moreover, the dynamic performance of different power generating unit in area-2 is also improved by the proposed AGC scheme, as visible by the total power generation response. As the additional load demand in area-1 is generated within the same area power generating unit, therefore the net power change in area- 2 is zero. The overall the system performance is evaluated based on cost function formulated as integral of square of errors. From Table 3, it is quite evident that the proposed AGC scheme improves the system performance over the conventional PID and model predictive controller.

\subsection{Robust Performance of Hybrid MPC-Based AGC Scheme.} The robustness of the proposed AGC scheme against parameters uncertainty is validated. In this case, all the time constant of power system generating sources, thermal, hydro, and DFIG are increased by $20 \%$ of nominal value, keeping the controller design at the nominal values. The same load disturbance of $2 \%$ in area-1 is considered for this study also. Figures 4(a) and 4(b) represent the dynamic response of frequency in area-1 and area-2, respectively. The tie-line power deviation is shown in Figure 4(c). From dynamic responses, it is quite clear that the fixed structure PID-based AGC scheme has larger deviation in peak overshoot and settling time for the variation in system parameters.

Nevertheless, the hybrid MPC-based AGC schemes handle the parameter uncertainties effectively and control the
TABLE 3: Performance index based on ISE

\begin{tabular}{lcc}
\hline PID & MPC & MPC+PID \\
\hline $4.355 \times 10^{-4}$ & $2.381 \times 10^{-4}$ & $1.497 \times 10^{-4}$ \\
\hline
\end{tabular}

system efficiently without losing the system stability. Moreover, the proposed AGC scheme enhances the system performance by achieving faster settling time with less peak overshoot and reduced oscillations as compared to model predictive frequency controller. The additional load demand in area- 1 is matched by the thermal, hydro, and wind generating units according to their share in the total power generation in that area.

The variation in system parameters oscillate thermal, hydro, and wind power generation around its operating point in case of conventional PID-based frequency regulator. However, MPC-based AGC scheme enhances the system performance as indicated in Table 4 , and the power output from all generating units is less oscillatory and quickly settles to a new steady-state condition. Since the disturbance has a more local impact, therefore the states of area-1 are disturbed more than the area- 2 and a similar trend in the peak overshoot, settling time, and oscillations is observed in the output power of thermal, hydro, and wind generating units as evident in Figures 4(d) and 4(e). The symbols and numerical data are given in Table 5.

\section{Discussion of Results}

The simulations presented in Section 4 show that the performance of PID is enhanced after MPC is hybridized with PID controller. The hybrid controller efficiently dampens the oscillation in system states after power system experience variation in states due to system operation or disturbance. Moreover, it is quite evident from the simulation study that PID can be replaced by the MPC for the AGC application, since it is controlling bandwidth and performance is better than the PID controller. Furthermore, the hybrid MPC +PID controller tightly damps the oscillation, which can be clearly observed in all the responses of the simulation. Lastly, the performance of the MPC depends on the prediction window, control window, and weights on states that should be selected optimally for the better results.

\section{Conclusion}

This paper presents hybrid MPC+PID-based AGC regulator for multisource power system model considering dominant participation of DFIG generating unit in frequency regulation. The decentralized MPC is designed for each area of the power system is based on local area system states. The integral of square of error is used for the performance evaluation of the proposed AGC scheme. The performance of the MPC depends on the prediction as well as the control interval selected. The simulations have also been carried out to validate the effectiveness of the proposed scheme for several varied parameters and load disturbances. A performance evaluation has been presented and discussed between 

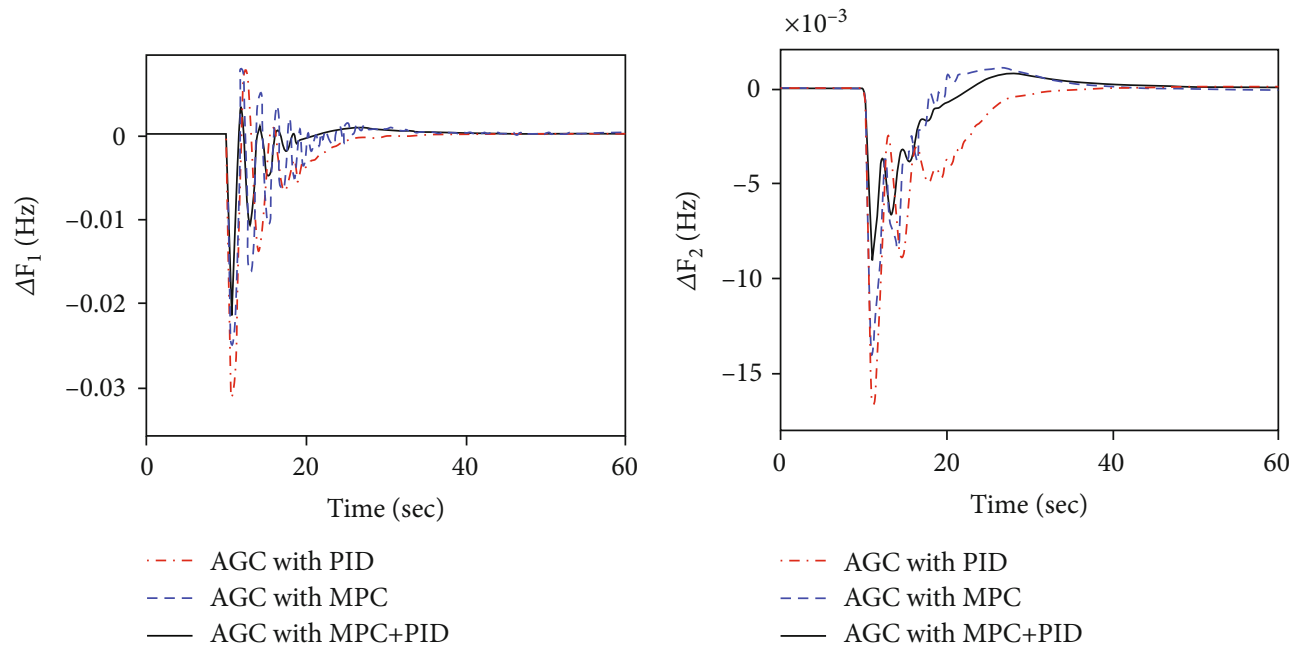

-. AGC with PID

- - - AGC with MPC

— AGC with MPC+PID

(a)

(b)
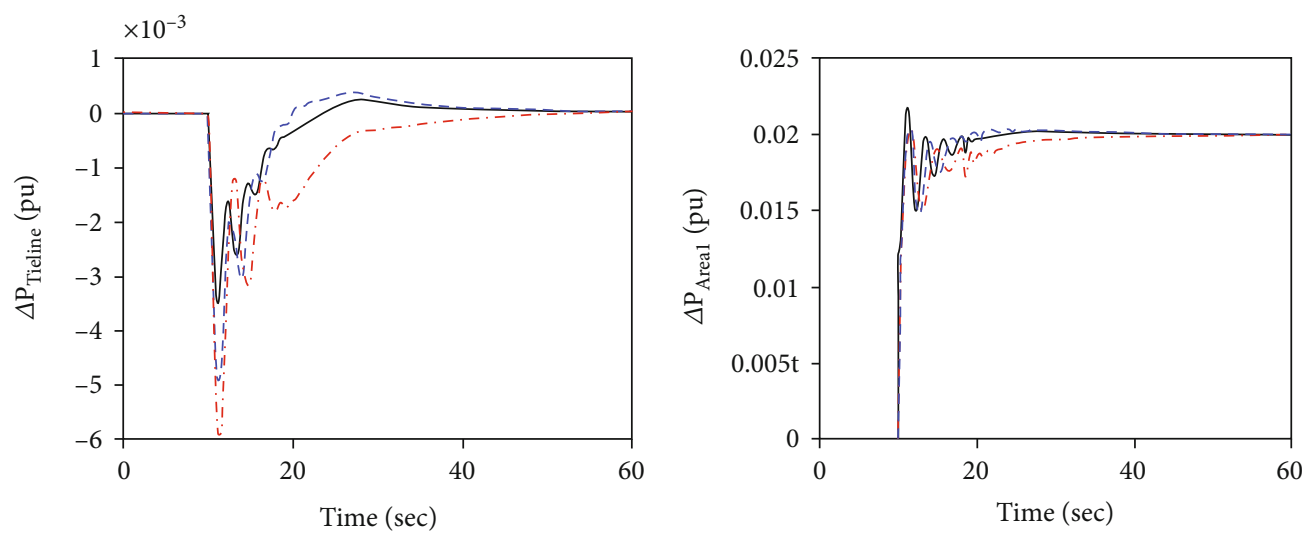

-. - AGC with PID

- - - AGC with MPC

— AGC with MPC+PID

-. - AGC with PID

- - AGC with MPC

— AGC with MPC+PID

(c)

(d)

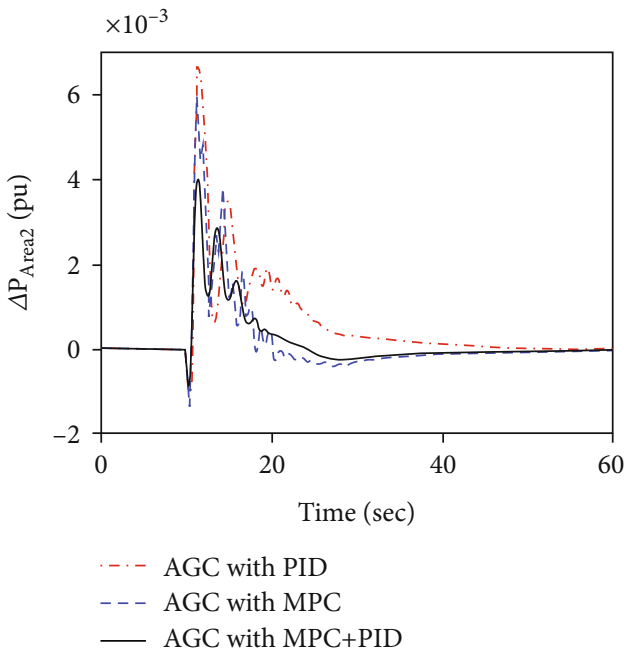

(e)

FIgure 4: Dynamic performance of multisource power system model for $2 \%$ load disturbance in area-1 and $20 \%$ parameter variation. (a) $\Delta F_{1}$ vs. time, (b) $\Delta F_{2}$ vs. time, (c) $\Delta P_{\text {tie }}$ vs. time, (d) $\Delta P_{\text {area-1 }}$ vs. time, and (e) $\Delta P_{\text {area }-2}$ vs. time. 
TABLE 4: Performance index based on ISE.

\begin{tabular}{lcc}
\hline PID & MPC & MPC+PID \\
\hline $5.015 \times 10^{-4}$ & $3.165 \times 10^{-4}$ & $1.728 \times 10^{-4}$ \\
\hline
\end{tabular}

TABLE 5: List of symbols and numerical data.

\begin{tabular}{|c|c|c|}
\hline Name of the parameters/term & $\begin{array}{l}\text { Symbol for } i^{\text {th }} \\
(i=1.2) \text { area }\end{array}$ & $\begin{array}{l}\text { Numerical } \\
\text { value }\end{array}$ \\
\hline Bias constant & $B_{i}$ & 0.4249 \\
\hline Regulation constant & $R_{i}$ & $2.4 \mathrm{~Hz} / \mathrm{puMW}$ \\
\hline $\begin{array}{l}\text { Steam turbine governor time } \\
\text { constant }\end{array}$ & $T_{g i}$ & $0.08 \mathrm{~s}$ \\
\hline Steam turbine time constant & $T_{t i}$ & $0.3 \mathrm{~s}$ \\
\hline $\begin{array}{l}\text { Coefficient of reheater steam } \\
\text { turbine }\end{array}$ & $K_{r i}$ & 0.3 \\
\hline $\begin{array}{l}\text { Steam turbine reheater time } \\
\text { constant }\end{array}$ & $T_{r i}$ & $10 \mathrm{~s}$ \\
\hline DFIG inertia & $H_{e}$ & $3.5 \mathrm{puMW} / \mathrm{s}$ \\
\hline Wind turbine regulation constant & $R_{w}$ & $3 \mathrm{~Hz} / \mathrm{puMW}$ \\
\hline $\begin{array}{l}\text { Frequency measurement } \\
\text { constant }\end{array}$ & $T_{r}$ & $0.1 \mathrm{~s}$ \\
\hline $\begin{array}{l}\text { Thermal power generation } \\
\text { contribution }\end{array}$ & $K_{t i}$ & 0.7 \\
\hline $\begin{array}{l}\text { Wind power generation } \\
\text { contribution }\end{array}$ & $K_{w i}$ & 0.1 \\
\hline Gain constant of power system & $K_{P i}$ & 120 \\
\hline Time constant of power system & $T_{P i}$ & $20 s$ \\
\hline Tie-line power coefficient & $T_{12}$ & $0.0868 \mathrm{MW}$ \\
\hline Inertia constant & $H$ & $\begin{array}{l}5 \mathrm{MW}-\mathrm{s} / \\
\mathrm{MVA}\end{array}$ \\
\hline Rated frequency & $F_{r}$ & $60 \mathrm{~Hz}$ \\
\hline Power rating of control area & $P_{r i}$ & $2000 \mathrm{MW}$ \\
\hline Second-order frequency stabilizer & $T_{1}$ & $0.121 \mathrm{~s}$ \\
\hline Second-order frequency stabilizer & $T_{2}$ & $0.80 \mathrm{~s}$ \\
\hline Second-order frequency stabilizer & $T_{3}$ & $0.011 \mathrm{~s}$ \\
\hline Second-order frequency stabilizer & $T_{4}$ & $0.148 \mathrm{~s}$ \\
\hline
\end{tabular}

the proposed AGC scheme and fixed structure conventional PID as well as MPC controllers. The simulation results and integral square performance criteria clearly revealed that the closed-loop system with hybrid MPC-based AGC scheme is quite robust against the parameter uncertainties and load perturbation as well as has appreciable performance compared to classical fixed structure PID and MPC-based AGC scheme. The limitation of the proposed work is the optimized sampling rate of the MPC so that each state is online solved with acceptable convergence criteria which shall be properly tuned with respect to type of disturbance incurred on the system. Moreover, the performance of the hybrid system will also degrade if the power system model becomes more complex such multiple distributed generation system is included in the calculation of area control error for the AGC controller.

\section{Nomenclature}

ACE: Area control error

ADP: Adaptive dynamic programming

AMPC: Adaptive model predictive controller

AGC: Automatic generation control

DFIG: Doubly fed induction generator

ETC: $\quad$ Event triggered control

GA: Genetic algorithm

GRC: Generation rate constraints

HPWH: Heat pump water heater

HTGA: Hybrid Taguchi-genetic algorithm

ISE: Integrated square error

LFC: Load frequency control

LQR: $\quad$ Linear quadratic regulator

MW: Mega watt

MVAR: Mega volt ampere reactive

MJOA: Modified Jaya optimization algorithm

MPC: $\quad$ Model predictive control

MOPSO: Multiobjective practical swarm optimization algorithm

P: $\quad$ Proportional

PI: $\quad$ Proportional integral

PID: $\quad$ Proportional integral derivative

RPME: Recursive polynomial model estimator

STC: $\quad$ Self-tuning control

UC: Unit commitment.

\section{Data Availability}

The data used to support the findings of this study are included within the article.

\section{Conflicts of Interest}

The authors declare that there are no competing interests regarding the publication of this paper.

\section{Acknowledgments}

The authors gratefully acknowledge Qassim University, represented by the Deanship of Scientific Research, on the financial support for this research under the number (5671-qec-2019-2-2-I) during the academic year $1440 \mathrm{AH} /$ 2019AD.

\section{References}

[1] M. Rouholamini, M. Rashidinejad, A. Abdollahi, and H. Ghasemnejad, "Frequency reserve within unit commitment considering spinning reserve uncertainty," International Journal of Energy Engineering, vol. 2, no. 4, pp. 177-183, 2012.

[2] J. F. Restrepo and F. D. Galiana, "Unit commitment with primary frequency regulation constraints," IEEE Transactions on Power Systems, vol. 20, no. 4, pp. 1836-1842, 2005.

[3] A. N. Venkat, I. A. Hiskens, J. B. Rawlings, and S. J. Wright, "Distributed MPC strategies with application to power system automatic generation control," IEEE Transactions on Control Systems Technology, vol. 16, no. 6, pp. 1192-1206, 2008. 
[4] M. Ma, H. Chen, X. Liu, and F. Allgöwer, "Distributed model predictive load frequency control of multi-area interconnected power system," International Journal of Electrical Power and Energy Systems, vol. 62, pp. 289-298, 2014.

[5] X. Liu, P. Guan, and C. W. Chan, "Nonlinear multivariable power plant coordinate control by constrained predictive scheme," IEEE Transactions on Control Systems Technology, vol. 18, no. 5, pp. 1116-1125, 2010.

[6] T. H. Mohamed, H. Bevrani, A. A. Hassan, and T. Hiyama, "Decentralized model predictive based load frequency control in an interconnected power system," Energy Conversion and Management, vol. 52, no. 2, pp. 1208-1214, 2011.

[7] T. H. Mohamed, J. Morel, H. Bevrani, and T. Hiyama, "Model predictive based load frequency control_design concerning wind turbines," International Journal of Electrical Power and Energy Systems, vol. 43, no. 1, pp. 859-867, 2012.

[8] M. Shiroei, A. M. Ranjbar, and T. Amraee, "A functional model predictive control approach for power system load frequency control considering generation rate constraint," International Transactions on Electrical Energy Systems, vol. 23, no. 2, pp. 214-229, 2013.

[9] M. Shiroei, M. R. Toulabi, and A. M. Ranjbar, "Robust multivariable predictive based load frequency control considering generation rate constraint," International Journal of Electrical Power \& Energy Systems, vol. 46, no. 1, pp. 405-413, 2013.

[10] M. A. Mohamed, A. A. Zaki Diab, H. Rezk, and T. Jin, “A novel adaptive model predictive controller for load frequency control of power systems integrated with DFIG wind turbines," Neural Computing and Applications, vol. 32, no. 11, pp. 7171-7181, 2020.

[11] H. Ye and Y. Liu, "Design of model predictive controllers for adaptive damping of inter-area oscillations," International Journal of Electrical Power and Energy Systems, vol. 45, no. 1, pp. 509-518, 2013.

[12] A. Oshnoei, R. Khezri, and S. M. Muyeen, "Model predictivebased secondary frequency control considering heat pump water heaters," Energies, vol. 12, no. 3, pp. 411-418, 2019.

[13] W. Qi, J. Liu, and P. D. Christofides, "Distributed supervisory predictive control of distributed wind and solar energy systems," IEEE Transactions on Control Systems Technology, vol. 21, no. 2, pp. 504-512, 2013.

[14] A. M. Ersdal, L. Imsland, and K. Uhlen, "Model predictive load-frequency control," IEEE Transactions on Power Systems, vol. 31, no. 1, pp. 777-785, 2016.

[15] X. Liu, X. Kong, and K. Y. Lee, “Distributed model predictive control for load frequency control with dynamic fuzzy valve position modelling for hydro-thermal power system," IET Control Theory \& Applications, vol. 10, no. 14, pp. 16531664, 2016.

[16] S. Kayalvizhi and D. M. Vinod Kumar, "Load frequency control of an isolated micro grid using fuzzy adaptive model predictive control," IEEE Access, vol. 5, pp. 16241-16251, 2017.

[17] M. S. Akbari, A. A. Safavi, N. Vafamand, T. Dragičević, and J. Rodriguez, "Fuzzy Mamdani-based model predictive load frequency control," in 2020 IEEE 11th International Symposium on Power Electronics for Distributed Generation Systems (PEDG), pp. 7-12, Dubrovnik, Croatia, 2020.

[18] C. Pradhan and C. N. Bhende, "Online load frequency control in wind integrated power systems using modified Jaya optimization," Engineering Applications of Artificial Intelligence, vol. 77, pp. 212-228, 2019.
[19] D. Rerkpreedapong, N. Atic, and A. Feliachi, "Economy oriented model predictive load frequency control," in Large Engineering Systems Conference on Power Engineering, 2003, pp. 12-16, Montreal, QC, Canada, 2003.

[20] L. C. Saikia, S. Mishra, N. Sinha, and J. Nanda, "Automatic generation control of a multi area hydrothermal system using reinforced learning neural network controller," International Journal of Electrical Power \& Energy Systems, vol. 33, no. 4, pp. 1101-1108, 2011.

[21] P. McNamara and F. Milano, "Model Predictive ControlBased AGC for Multi-Terminal HVDC-Connected AC grids," IEEE Transactions on Power Systems, vol. 33, no. 1, pp. 10361048, 2018.

[22] Z. Miao, L. Fan, D. Osborn, and S. Yuvarajan, "Control of DFIG-based wind generation to improve inter area oscillation damping," IEEE Transactions on Energy Conversion, vol. 24, no. 2, pp. 415-422, 2009.

[23] G. Ramtharan, N. Jenkins, and J. B. Ekanayake, "Frequency support from doubly fed induction generator wind turbines," IET Renewable Power Generation, vol. 1, no. 1, pp. 3-9, 2007.

[24] D. Peng, Y. Xu, and H. Zhao, "Research on intelligent predictive AGC of a thermal power unit based on control performance standards," Energies, vol. 12, pp. 1-23, 2019.

[25] A. B. Kunya, M. Argin, Y. Jibril, and Y. A. Shaaban, "Improved model predictive load frequency control of interconnected power system with synchronized automatic generation control loops," Beni-Suef University Journal of Basic and Applied Sciences, vol. 9, no. 1, pp. 1-13, 2020.

[26] N. Hasan, I. Nasirudin, and S. Farooq, "Hybrid Taguchi genetic algorithm-based AGC controller for multisource interconnected power system," Electric Power Components \& Systems, vol. 47, no. 1-2, pp. 101-112, 2019.

[27] L. Dong, Y. Tang, H. He, and C. Sun, "An event-triggered approach for load frequency control with supplementary ADP," IEEE Transactions on Power Systems, vol. 32, no. 1, pp. 581-589, 2017.

[28] A. Elgammal and T. Ramlal, "Optimal frequency control management of grid integration PV/wind/FC/storage battery based smart grid using multi objective particle swarm optimization (MOPSO) and model predictive control (MPC)," European Journal of Engineering and Technology Research, vol. 6, no. 5, pp. 50-56, 2021.

[29] D. Bhagdev, R. Mandal, and K. Chatterjee, "Study and application of MPC for AGC of two area interconnected thermalhydro-wind system," in IEEE Conf. proceeding Innovations in Power and Advanced Computing Technologies (i-PACT), Vellore, India, March 2019.

[30] S. Mishra, P. C. Nayak, U. C. Prusty, and R. C. Prusty, "Model predictive controller based load frequency control of isolated microgrid system integrated to plugged-in electric vehicle," in 1st Odisha International Conference on Electrical Power Engineering, Communication and Computing Technology $(\mathrm{O}-$ DICON), India, January 2021.

[31] R. G. George, H. M. Hasanien, A. Al-Durra, and M. Abd-latiff Badr, "Model predictive controller for performance enhancement of automatic voltage regulator system," International Journal on Energy Conversion, vol. 6, no. 6, 2018. 